\title{
PREVALENCIA DE COMPRA SIN RECETA Y RECOMENDACIÓN DE ANTIBIÓTICOS PARA NIÑOS MENORES DE 5 AÑOS EN FARMACIAS PRIVADAS DE ZONAS PERIURBANAS EN LIMA, PERÚ
}

\author{
Lucie Ecker ${ }^{1,2, a}$, Joaquín Ruiz,3,b, Martha Vargas ${ }^{4, c}$, Luis J. Del Valle ${ }^{5, c}$, Theresa J. Ochoa ${ }^{2,6, d}$
}

\begin{abstract}
RESUMEN
Objetivos. Determinar la prevalencia de compra sin receta médica y recomendación de antibióticos para su uso en niños menores de 5 años en farmacias privadas de zonas periurbanas de Lima. Materiales y métodos. Se aplicó una encuesta anónima poblacional en adultos que compraron un antibiótico para un niño menor de cinco años en una farmacia privada. En un subgrupo de farmacias se aplicaron casos ficticios para evaluar prácticas de recomendación de antibióticos. Resultados. La prevalencia de compra sin receta fue de 13\%; 1,7\% por automedicación y 11,3\% por indicación del farmacéutico. El $66,7 \%$ de los medicamentos para tratar resfrío fueron antibióticos de los cuales $56,9 \%$ tenían receta. El $64,4 \%$ de los medicamentos comprados para tratar broncoespasmo fueron antibióticos; el $96,4 \%$ de los medicamentos para tratar diarreas acuosas y el $90,9 \%$ de los medicamentos para tratar faringitis. Amoxicilina $(51,5 \%)$ y trimetoprimsulfametoxazol $(20,6 \%)$ fueron los más comprados. En el $60 \%$ de los casos ficticios de resfrío común, en el $76 \%$ de los de broncoespasmo, en el $44 \%$ de los de diarrea acuosa y en el $76 \%$ de los casos de disentería, el farmacéutico indicó un antibiótico. Conclusiones. La compra de antibióticos para niños menores de cinco años en farmacias privadas es principalmente con receta médica. La recomendación de antibióticos por parte de la farmacia es alta cuando no hay receta médica. Urgen medidas para disminuir el uso inadecuado de antibióticos tanto a nivel médico como en las farmacias.
\end{abstract}

Palabras clave: Mal uso de medicamentos de venta con receta; Farmacoresistencia microbiana; Niño; Prescripción de medicamentos; Farmacias. (fuente: DeCS BIREME)

\section{PREVALENCE OF PURCHASE OF ANTIBIOTICS WITHOUT PRESCRIPTION AND ANTIBIOTIC RECOMMENDATION PRACTICES FOR CHILDREN UNDER FIVE YEARS OF AGE IN PRIVATE PHARMACIES IN PERI-URBAN AREAS OF LIMA, PERU}

\begin{abstract}
Objectives. To determine the prevalence of purchase of antibiotics without prescription and antibiotic recommendation for its use in children $<5$ years of age in private pharmacies in peri-urban areas of Lima. Materials and methods. An anonymous population survey was conducted among adults who bought an antibiotic for a child $<5$ years of age from a private pharmacy. Fictitious cases were applied in a subgroup of pharmacies to evaluate antibiotic recommendation practices. Results. The prevalence of purchase of antibiotics without a prescription was $13 \%$, including $1.7 \%$ that were chosen by consumers and $11.3 \%$ that were recommended by pharmacists. A total of $66.7 \%$ of the medications purchased to treat colds were antibiotics, $56.9 \%$ of which were prescribed. $64.4 \%$ of medications purchased to treat bronchospasm, $96.4 \%$ of the medications to treat watery diarrhea and $90.9 \%$ of the medications to treat pharyngitis were antibiotics. Amoxicillin $(51.5 \%)$ and trimethoprimsulfamethoxazole $(20.6 \%)$ were the most commonly purchased antibiotics. A pharmacist suggested an antibiotic in $60 \%$ of the fictitious common cold cases, $76 \%$ of the bronchospasm cases, $44 \%$ of the watery diarrhea cases, and $76 \%$ of the dysentery cases. Conclusions. The purchase of antibiotics for children $<5$ years of age in private pharmacies primarily occurs with a medical prescription. The pharmacist commonly recommended an antibiotic in cases lacking a prescription. Measures are needed to reduce the inappropriate use of antibiotics at medical facilities and in pharmacies.
\end{abstract}

Key words: Prescription drug misuse, Drug resistance, microbial; child ; Drug prescription; Pharmacies (source: MeSH NLM)

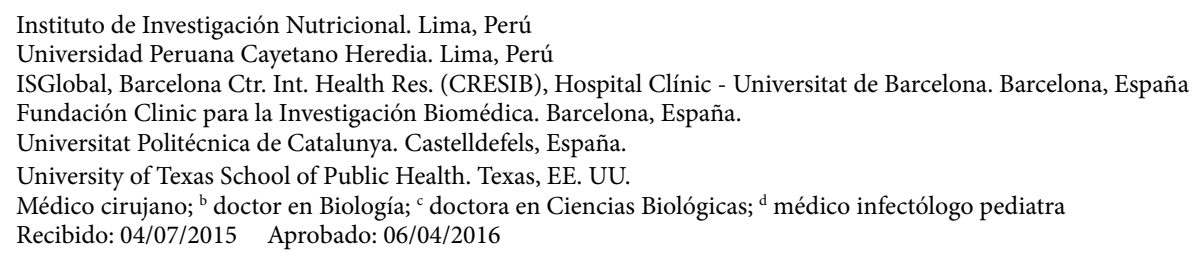

Citar como: Ecker L, Ruiz J, Vargas M, Del Valle LJ, Ochoa TJ. Prevalencia de compra sin receta y recomendación de antibióticos para niños menores de 5 años en farmacias privadas de zonas periurbanas en Lima, Perú. Rev Peru Med Exp Salud Publica. 2016;33(2):215-23. doi: 10.17843/rpmesp.2016.332.2152 


\section{INTRODUCCIÓN}

Los antibióticos han disminuido notablemente la morbimortalidad por diversas enfermedades $y$, actualmente, son las drogas más usadas en niños, en especial en infecciones respiratorias y diarrea ${ }^{(1-5)}$. Con el aumento de su uso también ha surgido el aumento de la resistencia antibiótica de los patógenos adquiridos en la comunidad, considerándose un problema creciente a nivel mundial que requiere una pronta intervención ${ }^{(6-9)}$. Las bacterias multirresistentes son consideradas como una enfermedad emergente global y un problema mayor de salud pública ${ }^{(10)}$. El Perú es uno de los países de Latinoamérica en el que más se ha incrementado el uso de antimicrobianos (11) y es uno de los principales factores por el cual la resistencia también ha venido en aumento. Diversos patógenos y bacterias comensales presentan altos niveles de resistencia ${ }^{(12-15)}$.

En países en vías de desarrollo, la automedicación ha sido reconocida como una de las causas principales del uso inapropiado de antibióticos (16,17). Actualmente, en muchos países de bajos ingresos, los antibióticos son comúnmente adquiridos en establecimientos privados de venta de medicamentos ${ }^{(18,19)}$. Las boticas o farmacias se han convertido en importantes proveedores de consejos de salud, evitando así la larga espera y los costos de una atención médica para el tratamiento de enfermedades de leve intensidad, en su mayoría ${ }^{(20)}$. La amplia disponibilidad de antibióticos hace que lleguen a sitios más remotos en donde antes era escasa y se puedan tratar infecciones en personas que antes no se trataban.

Si bien en Perú existe la norma de no vender antimicrobianos sin receta médica, esto no se cumple completamente, pudiendo adquirirse un antibiótico sin receta médica en la mayoría de los establecimientos de venta privados. Por ello es necesario más medidas que la sola regulación de la venta sin receta, ya que se ha demostrado en estudios que en la mayoría de los casos la prescripción es inadecuada, y que en el caso de niños pequeños los padres prefieren no automedicar ${ }^{(1-3)}$. En Perú no se han realizado suficientes intervenciones para disminuir el uso indiscriminado de antibióticos, por lo que es necesario tener datos para poder establecer adecuadas medidas para su uso adecuado.

Existe poca información acerca de las prácticas de recomendación de antimicrobianos sin prescripción médica para niños menores de 5 años, por parte del personal de farmacias privadas cercanas a los centros de atención primaria en zonas periurbanas de Lima, Perú. Asimismo, se desconoce la prevalencia de compra de antibióticos sin prescripción, en niños menores de 5 años, lo que no permite tener la visión global del problema para poder establecer las pautas de intervención adecuadas para disminuir el uso inadecuado de antibióticos y así poder alterar los patrones de resistencia.

El objetivo de este estudio es determinar la prevalencia de compra de antibióticos sin prescripción para su uso en niños menores de 5 años en boticas y farmacias privadas, así como determinar las prácticas de recomendación de antibióticos del personal de farmacias privadas cercanas a los centros de atención primaria en zonas periurbanas de Lima, Perú.

\section{MATERIALES Y MÉTODOS}

\section{DISEÑO}

Se realizó un estudio de tipo transversal aplicando una encuesta poblacional a usuarios adultos de farmacias privadas que hayan comprado un medicamento para un niño menor de cinco años en una farmacia o botica privada cercana a un centro de salud de la Red de Salud de San Juan de Lurigancho (solo se consideraron boticas y farmacias privadas, dado que en las farmacias de los establecimientos de salud del Ministerio de Salud es requisito indispensable presentar la receta médica). Mediante los registros de la red de salud se incluyeron a todos los establecimientos de salud de atención primaria y se buscaron, mediante un censo, las farmacias y boticas circundantes a estos. La zona se escogió por conveniencia por ser parte de los estudios anteriores de uso de antibióticos. El estudio se realizó entre agosto y diciembre del año 2012.

\section{CÁLCULO DEL TAMAÑO MUESTRAL}

Teniendo en cuenta que la Red San Juan de Lurigancho tiene una población de niños menores de cinco año estimada de 84569 (según la Red San Juan de Lurigancho http://www. redsasjl.gob.pe/) y en base al estudio previo (3), alrededor de $10 \%$ (8457) de esta población se encuentra usando un antibiótico por alguna enfermedad y esperando una prevalencia de uso de antibióticos sin prescripción del $20 \%$, con un nivel de confianza de $95 \%$ y con un $10 \%$ adicional por efecto de diseño o rechazo al estudio, se calculó un tamaño muestral de 263 usuarios adultos que hayan comprado un antibiótico para un niño menor de 5 años en una farmacia o botica particular. Tomando en cuenta las posibles faltas de respuesta este número se incrementó a 293 encuestas. El cálculo se realizó con el programa de libre acceso en la web openepi (http://www.openepi.com).

\section{ENCUESTA}

Previo estudio piloto del cuestionario por emplear, se aplicó la encuesta en farmacias o boticas que se encontraban en un radio no mayor a los $500 \mathrm{~m}$ del centro de salud. De estas, se seleccionó la farmacia con mayor afluencia cercana a cada centro de atención primaria de la salud de la red San Juan de Lurigancho y se realizó la 
encuesta durante un día, cambiando a otra ubicación al día siguiente. Se realizaron las encuestas en todos los usuarios adultos que compraron un medicamento para un niño menor de 5 años, hasta alcanzar 293 encuestas de usuarios adultos que habían comprado un antibiótico para un niño menor de 5 años.

Se realizaron encuestas en estos establecimientos en dos horarios de atención: mañana (8.00 a 14.00 h) y tarde (14.00 a $20.00 \mathrm{~h}$ ) en dos días, uno durante la semana y otro durante el fin de semana en las farmacias seleccionadas, hasta alcanzar la muestra requerida.

Las encuestas fueron estructuradas, se aplicaron a la salida de las farmacias y fue anónima y voluntaria, por lo que la confidencialidad de los participantes no se vio afectada. Para determinar si se trataba de un usuario que compró un medicamento para un niño menor de cinco años, se aplicó un tamizaje rápido que constó de una pregunta sobre la compra de un medicamento para un niño menor de cinco años. Si la respuesta era positiva se iniciaba el cuestionario completo.

Asimismo, se efectuó un estudio exploratorio en un subgrupo de 100 farmacias escogidas al azar, que constó de cuatro casos diferentes de pacientes ficticios en niños menores de cinco años, sin receta médica, que fueron elaborados por médicos pediatras y presentados por las trabajadoras al personal de farmacia, para evaluar sus prácticas de recomendación de antibióticos. En total, se realizaron 25 simulaciones de cada caso. Los casos que se incluyeron fueron diarrea acuosa, resfrío común, broncoespasmo y disentería.

\section{ANÁLISIS}

Para determinar la prevalencia de compra sin receta, solo se tomaron los datos de los usuarios que compraron un antibiótico. La prevalencia de compra de antibióticos sin receta médica se calculó con un nivel de confianza de $95 \%$, usando como denominador al número de medicamentos comprados para niños menores de 5 años. Como numerador se usó el número de usuarios adultos que compra un antibiótico en una farmacia o botica particular sin prescripción médica para su uso en un niño menor de cinco años de edad. Para determinar el diagnóstico por el cual compraron el medicamento se corroboraron las recetas médicas, si es que las tenían y si es que había en ellas el diagnóstico, de lo contrario, encuestadores previamente capacitados preguntaron por los signos y síntomas que presentaban los niños. Esto fue analizado posteriormente por un médico general con asesoría de un médico pediatra, quienes determinaron los diagnósticos probables. Se analizaron las causas referidas por el entrevistado, por las cuales habían comprado medicamentos sin receta médica.
Se realizó el análisis descriptivo de las prácticas de recomendación de antibióticos por parte de los farmacéuticos que se evaluaron en los casos ficticios.

Se realizó el análisis exploratorio de los factores que podrían estar asociados al uso de antibióticos sin receta médica en niños menores de cinco años. Este análisis se hizo mediante regresión logística. La variable dependiente fue el uso de antibióticos en niños menores de cinco años sin receta médica. Las variables que se exploraron fueron edad del cuidador, sexo del cuidador, edad del niño y parentesco con el niño. Los resultados se reportan como odds ratios (OR) con intervalos de confianza al 95\% (IC 95\%) y un nivel de significancia de $p<0,05$. Se usó STATA 11 para el análisis.

\section{CONSIDERACIONES ÉTICAS}

El estudio fue presentado y aprobado por el Comité de Ética de la Universidad Peruana Cayetano Heredia en Lima, Perú. Antes de administrar el cuestionario, se obtuvo el consentimiento verbal de los encuestados. No se incluyeron identificadores personales en la encuesta.

\section{RESULTADOS}

Se realizaron un total de 474 encuestas hasta alcanzar 293 encuestas en cuidadores que habían comprado un antibiótico para un niño menor de 5 años. Ocho cuidadores compraron dos antibióticos para su niño, por lo que en total se compraron 301 antibióticos. Se abarcaron farmacias de 31 de los 35 establecimientos de salud de la zona, por haber alcanzado el tamaño muestral adecuado. No hubo rechazos a la encuesta.

Las personas que compraron un medicamento para un niño de 5 años fueron principalmente su madre $(85,7 \%)$, en un $6,5 \%$ su abuela o abuelo y en $3,8 \%$ el padre. El $3 \%$ de los compradores tenía otra relación con el niño y $8(1 \%)$ personas no refirieron su relación. La mediana de edad de los cuidadores fue de 29 años variando en un rango de 15 a 75 años.

De los 474 casos en niños menores de 5 años para los que se compró un medicamento se encontraron $204(43 \%)$ casos de resfrío común, 90 (19\%) casos de broncoespasmo, $56(11,8 \%)$ casos de diarrea acuosa y $33(7 \%)$ casos de faringitis (Tabla 1$)$.

De los medicamentos comprados 293 (61,8\%) fueron un antibiótico (IC 95\%: 57\%-66\%). De las personas que compraron un antibiótico 255 (87\%) lo compraron por indicación médica (IC 95\%: 83\%-91\%). El 45,5\% (IC 95\%: 39\%-52\%) de los antibióticos recetados fueron para tratar resfríos, 21,6\% (IC 95\%:17\%-27\%) parar tratar broncoespasmos, 16,5\% (IC 95\%:12\%-22\%) para tratar diarrea acuosa y 10,2\% (IC 95\%: 7\%-15\%) para tratar faringitis (Tabla 1). 
Tabla 1. Porcentaje de medicamentos comprados según el tipo de prescripción y diagnóstico

\begin{tabular}{|c|c|c|c|c|c|c|c|c|c|}
\hline \multirow{3}{*}{ Diagnósticos } & \multicolumn{4}{|c|}{ Compraron otro medicamento } & \multicolumn{4}{|c|}{ Compraron antibióticos } & \multirow{2}{*}{$\begin{array}{c}\text { Total } \\
\text { ambos }\end{array}$} \\
\hline & $\begin{array}{l}\text { Recetó el } \\
\text { médico }\end{array}$ & $\begin{array}{l}\text { Recomendó } \\
\text { farmacéutico }\end{array}$ & Automedicó & Total & $\begin{array}{l}\text { Recetó el } \\
\text { médico }\end{array}$ & $\begin{array}{l}\text { Recomendó } \\
\text { farmacéutico }\end{array}$ & $\begin{array}{l}\text { Autome- } \\
\text { dicación }\end{array}$ & Total & \\
\hline & $(n=130)$ & $(n=28)$ & $(n=23)$ & $(\mathrm{N}=181)$ & $(n=255)$ & $(n=33)$ & $(n=5)$ & $(\mathrm{N}=293)$ & $(\mathrm{N}=474)$ \\
\hline Resfrío común & 32,3 & 57,1 & 43,5 & 37,6 & 45,5 & 51,5 & 60,0 & 46,6 & 43,0 \\
\hline Broncoespasmo & 22,3 & 3,6 & 8,7 & 17,7 & 21,6 & 6,1 & 20,0 & 19,9 & 19,0 \\
\hline Diarrea acuosa & 1,5 & 7,1 & 4,3 & 2,8 & 16,5 & 27,3 & 0 & 17,5 & 11,8 \\
\hline Faringitis & 1,5 & 0 & 4,3 & 1,7 & 10,2 & 12,1 & 0 & 10,3 & 7,0 \\
\hline Infección dérmica & 0 & 3,6 & 0 & 0,6 & 2,4 & 0 & 20,0 & 2,4 & 1,7 \\
\hline Disentería & 0 & 0 & 0 & 0 & 2 & 3 & 0,0 & 2,1 & 1,3 \\
\hline Otitis & 0 & 0 & 0 & 0 & 0,8 & 0,0 & 0,0 & 0,7 & 0,4 \\
\hline Infección urinaria & 0 & 0 & 0 & 0 & 0,8 & 0 & 0 & 0,7 & 0,4 \\
\hline Neumonía & 0 & 0 & 0 & 0 & 0,4 & 0 & 0 & 0,3 & 0,2 \\
\hline Fiebre & 9,2 & 14,3 & 13 & 10,5 & 0 & 0 & 0 & 0 & 4,0 \\
\hline Anemia & 10 & 0 & 4,3 & 7,7 & 0 & 0 & 0 & 0 & 3,0 \\
\hline Alergia & 6,9 & 7,1 & 4,3 & 6,6 & 0 & 0 & 0 & 0 & 2,5 \\
\hline Dermatitis & 5,4 & 3,6 & 0 & 4,4 & 0 & 0 & 0 & 0 & 1,7 \\
\hline Conjuntivitis & 0,8 & 0 & 0 & 0,6 & 0 & 0 & 0 & 0 & 0,2 \\
\hline Otros* & 10 & 3,6 & 17,4 & 9,9 & 0 & 0 & 0 & 0 & 3,8 \\
\hline
\end{tabular}

* Se incluyeron diagnósticos acarosis, aftas orales, candidiasis oral, cardiopatía congénita, cólico de gases, contusión, estreñimiento, hemangioma, niño sano (vacuna), parasitosis.

La prevalencia de compra de antibióticos sin receta médica fue de 13\%; por sugerencia del farmacéutico $11,3 \%$, y por automedicación 1,7\%. De los cuidadores que recibieron una recomendación del farmacéutico solamente $42,4 \%$ recibieron consejo de visitar un médico ante algún signo de alarma. El $60,6 \%$ de los farmacéuticos que recomendaron un antibiótico no preguntaron si el niño era alérgico a algún medicamento.

Amoxicilina fue el antibiótico mayormente comprado $(51,5 \%)$, seguido por trimetoprim-sulfametoxazol (TMP-SMX) (20,6\%). Los únicos antibióticos que se compraron sin receta médica fueron amoxicilina, TMP-SMX, amoxicilina-ácido clalvulánico, penicilina y furazolidona (Figura 1).

De los antibióticos comprados para tratar resfrío común, amoxicilina fue el que se compró con mayor frecuencia $(72,7 \%) ; 63,3 \%$ recetado por el médico. El antibiótico comprado con mayor frecuencia para tratar broncoespasmo también fue amoxicilina (58,6\%), $56,9 \%$ recetado por el médico. Para diarrea acuosa se compró preferentemente TMP-SMX (53,7\%), 42,6\% recetados por el médico. Para tratar faringitis se compró principalmente amoxicilina (46,9\%); 37,5\% con receta médica (Figura 2).
Resfrío común fue el diagnóstico por el que se compraron la mayor cantidad de antibióticos $(46,6 \%)$ seguido de broncoespasmo (19,9\%) y diarrea acuosa (17,5\%). El $51,5 \%$ de los antibióticos recomendados por el farmacéutico fueron para tratar resfrío común, 27,3\% para tratar diarrea acuosa. Los antibióticos comprados por automedicación fueron para tratar resfrío común $(60 \%)$, broncoespasmo (20\%) e infección dérmica (20\%). Los medicamentos que no eran antibióticos comprados también fueron en su mayoría para tratar resfrío común $(37,6 \%)$ y en segundo lugar para tratar broncoespasmo $(17,7 \%)$ (Tabla 1$)$.

Analizando por diagnóstico, el $87 \%$ de los antibióticos comprados para todos los diagnósticos fueron prescritos por un médico (Tabla 2).

El $66,7 \%$ de los medicamentos comprados para tratar resfrío común fueron antibióticos, de los cuales $56,9 \%$ fueron prescritos por el médico. El $64,4 \%$ de los medicamentos comprados para tratar broncoespasmo fueron antibióticos; $61,1 \%$ indicados por médicos. El $96,4 \%$ de los medicamentos comprados para tratar diarreas acuosas fueron antibióticos, de estos, 80,4\% fueron prescritos por un médico. El 90,9\% de los medicamentos comprados para tratar faringitis fueron antibiótico, $81,8 \%$ fueron indicados por el médico (Tabla 3). 


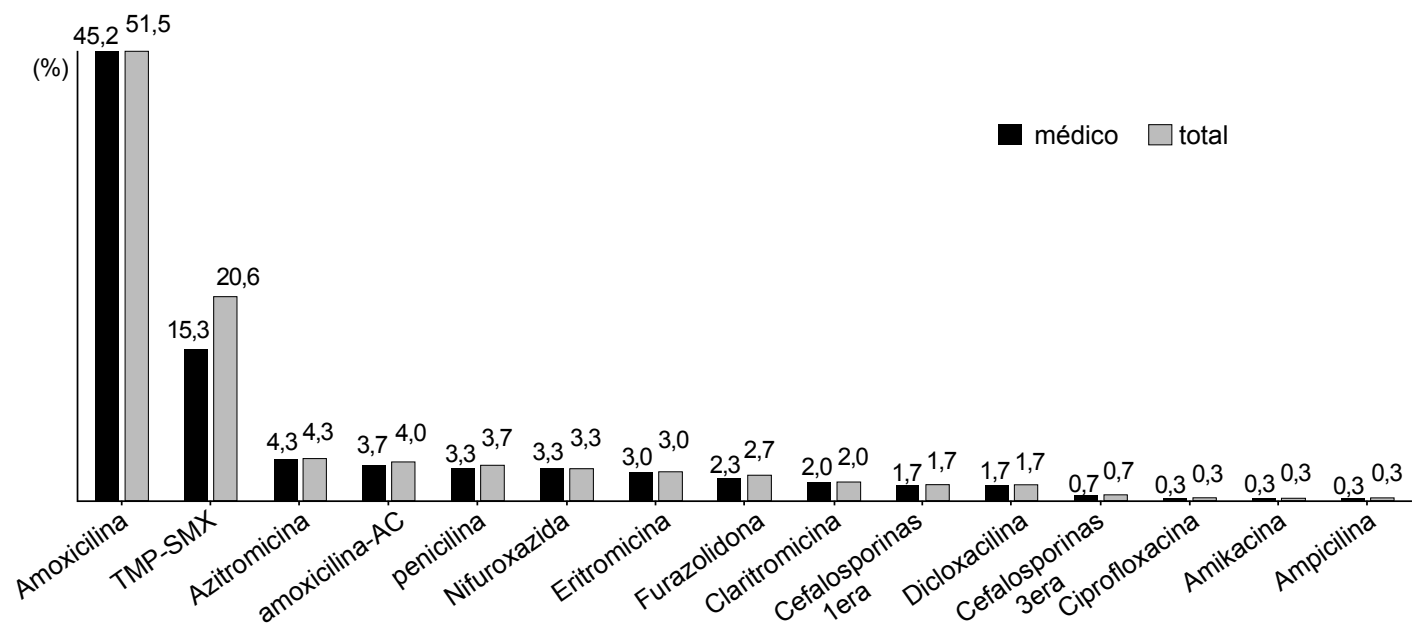

Figura 1. Porcentaje de antibióticos comprados

*Total de prescripciones: 301.

Se compraron antipiréticos para el $41,7 \%$ de los resfríos, para el $44,6 \%$ de las diarreas y para el $45,5 \%$ de las faringitis. Para el $17,9 \%$ de las diarreas se compraron probióticos (Tabla 3).

En el análisis bivariado de los factores asociados al uso de antibióticos sin receta médica ninguno tuvo significancia estadística.

Los casos ficticios se aplicaron en 100 farmacias; 25 casos de resfrío común, 25 casos de broncoespasmo, 25 casos de disentería y 25 casos de diarrea acuosa.
En el $60 \%$ de los casos ficticios de resfrío común, el farmacéutico indicó un antibiótico, en el $36 \%$ un sintomático y solo $4 \%$ recomendaron acudir a un médico (Figura 3). Los antibióticos indicados fueron amoxicilina $66,7 \%$, TMP-SMX $(26,7 \%$ ) y cefalexina $(6,7 \%)$. En cuanto a los casos ficticios de broncoespasmo, en el $76 \%$ los farmacéuticos recomendaron buscar atención médica, mientras que 16\% indicaron un antibiótico (Figura 3). Los antibióticos indicados para tratar broncoespasmo fueron amoxicilina (75\%) y TMP-SMX (25\%). En el $44 \%$ de los casos de diarrea acuosa el farmacéutico recomendó un antibiótico, en el 32\% recomendó

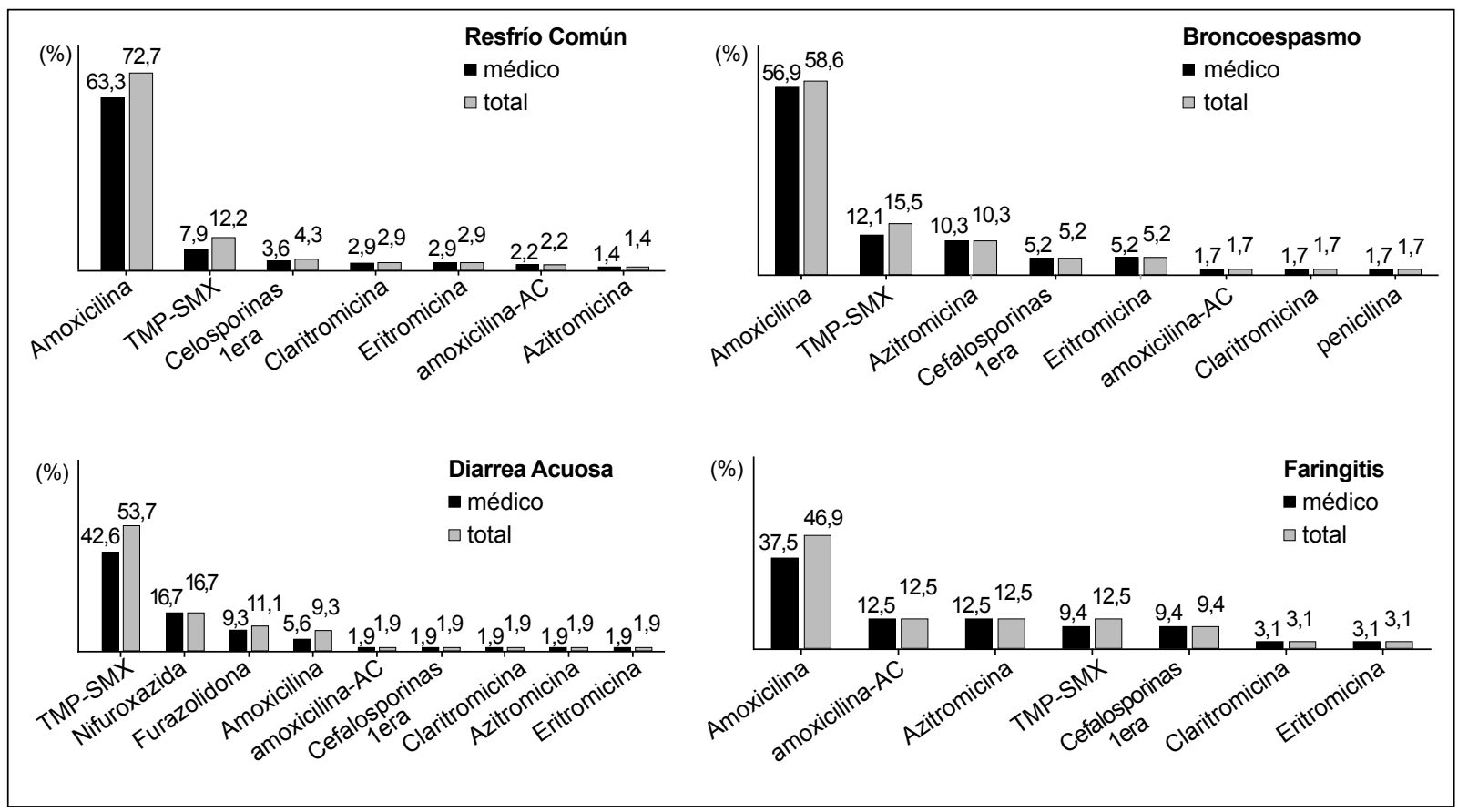

Figura 2.Tipo de antibióticos comprados e indicados según diagnóstico 
Tabla 2. Porcentaje de antibióticos comprados según tipo de prescripción y diagnóstico

\begin{tabular}{|c|c|c|c|c|}
\hline \multirow[t]{2}{*}{ Diagnósticos } & Total & Recetó el médico & $\begin{array}{l}\text { Recomendó } \\
\text { farmacéutico }\end{array}$ & Automedicó \\
\hline & $(n=293)$ & $(n=255)$ & $(n=33)$ & $(n=5)$ \\
\hline Resfrío común & 46,4 & 85,3 & 0,5 & 2,2 \\
\hline Broncoespasmo & 19,8 & 94,8 & 0,0 & 1,7 \\
\hline Diarrea acuosa & 17,4 & 82,4 & 0,0 & 0,0 \\
\hline Faringitis & 10,2 & 86,7 & 0,0 & 0,0 \\
\hline Infección dérmica & 2,4 & 85,7 & 0,4 & 14,3 \\
\hline Disentería & 2,0 & 83,3 & 0,0 & 0,0 \\
\hline Otitis & 0,7 & 100,0 & 0,0 & 0,0 \\
\hline Infección urinaria & 0,7 & 100,0 & 0,0 & 0,0 \\
\hline Neumonía & 0,3 & 100,0 & 0,0 & 0,0 \\
\hline
\end{tabular}

buscar consejo médico (Figura 3). Los antibióticos más recomendados fueron TMP-SMX $(45,5 \%)$ y furazolidona $(36,4 \%)$. En los casos de disentería se recomendaron antibióticos en el $76 \%$ de los casos, mientras que solo en $20 \%$ se recomendó buscar ayuda médica (Figura 3 ). Los antibióticos mayormente recomendados para tratar disentería fueron TMP-SMX (78,9\%) y furazolidona $(15,8 \%)$.

El 25,8\% de los que compraron cualquier medicamento sin receta médica dieron la razón por la cual lo habían hecho. Solo $13,2 \%$ de los que compraron antibióticos sin receta médica para su niño menor de 5 años dieron la razón por la cual lo habían hecho. La mayoría que compró sin receta médica, lo hizo porque la atención médica demora mucho $(21,3 \%)$ y porque creían que se trataba de un caso leve y no era necesario llevarlo al médico (18\%). Las principales causas por la que compraron antibióticos sin receta médica fueron la demora en la atención médica $(28,9 \%)$ y porque no había acceso a atención a esa hora (18,4\%) (Figura 4).

\section{DISCUSIÓN}

El uso excesivo de antibióticos es uno de los principales factores que contribuyen a la aparición de la resistencia a los antimicrobianos que, al aumentar, reduce la posibilidad de un tratamiento efectivo de las infecciones con los medicamentos existentes, se prolonga el tiempo de enfermedad y se aumenta el costo al tener que utilizar alternativas terapéuticas ${ }^{(10)}$.

En un estudio de tendencias de uso de antibióticos en América Latina entre los años 1997 y 2007, el Perú mostró el mayor incremento en el uso de antibióticos $(70,6 \%)$, otros países que incrementaron su uso fueron Uruguay en segundo lugar (64\%) y Venezuela $(43 \%)$, mientras que Colombia y Chile mostraron una disminución en el uso ${ }^{(11)}$. Asimismo, el mismo estudio demuestra que el uso de macrólidos, lincosamidas y streptograminas se ha incrementado ampliamente en el Perú $(82 \%)$, Brasil $(61,5 \%)$ y Argentina $(42 \%){ }^{(11)}$. El

Tabla 3. Porcentaje de medicamentos comprados según diagnóstico

\begin{tabular}{|c|c|c|c|c|c|c|c|c|}
\hline \multirow{2}{*}{ Medicamentos } & \multicolumn{2}{|c|}{$\begin{array}{l}\text { Resfrío común } \\
(n=204)\end{array}$} & \multicolumn{2}{|c|}{$\begin{array}{c}\begin{array}{c}\text { Broncoespasmo } \\
(n=90)\end{array} \\
\end{array}$} & \multicolumn{2}{|c|}{$\begin{array}{c}\text { Diarrea acuosa } \\
(n=56)\end{array}$} & \multicolumn{2}{|c|}{$\begin{array}{c}\text { Faringítis } \\
(n=33)\end{array}$} \\
\hline & $\begin{array}{l}\text { Indicó el } \\
\text { médico }\end{array}$ & Total & $\begin{array}{l}\text { Indicó el } \\
\text { médico }\end{array}$ & Total & $\begin{array}{l}\text { Indicó el } \\
\text { médico }\end{array}$ & Total & $\begin{array}{l}\text { Indicó el } \\
\text { médico }\end{array}$ & Total \\
\hline Antibióticos & 56,9 & 66,7 & 61,1 & 64,4 & 80,4 & 96,4 & 81,8 & 90,9 \\
\hline Antipiréticos & 32,8 & 41,7 & 11,1 & 13,3 & 35,7 & 44,6 & 36,4 & 45,5 \\
\hline Suero fisiológico & 2,0 & 2,0 & 2,2 & 2,2 & 3,6 & 5,4 & 0,0 & 0,0 \\
\hline Probióticos & 3,9 & 3,9 & 5,6 & 5,6 & 16,1 & 17,9 & 0,0 & 3,0 \\
\hline Medicina natural & 0,0 & 0,0 & 0,0 & 0,0 & 7,1 & 8,9 & 0,0 & 0,0 \\
\hline Mucolíticos - expectorantes & 1,0 & 3,4 & 5,6 & 6,7 & 0,0 & 0,0 & 0,0 & 0,0 \\
\hline Antihistamínicos & 9,3 & 16,7 & 15,6 & 16,7 & 0,0 & 0,0 & 0,0 & 0,0 \\
\hline Antigripales & 15,7 & 16,7 & 18,9 & 20,0 & 0,0 & 0,0 & 6,1 & 6,1 \\
\hline Corticoides & 2,0 & 4,9 & 20,0 & 20,0 & 0,0 & 0,0 & 0,0 & 0,0 \\
\hline Broncodilatadores & 4,4 & 5,4 & 41,1 & 43,3 & 0,0 & 0,0 & 6,1 & 6,1 \\
\hline
\end{tabular}




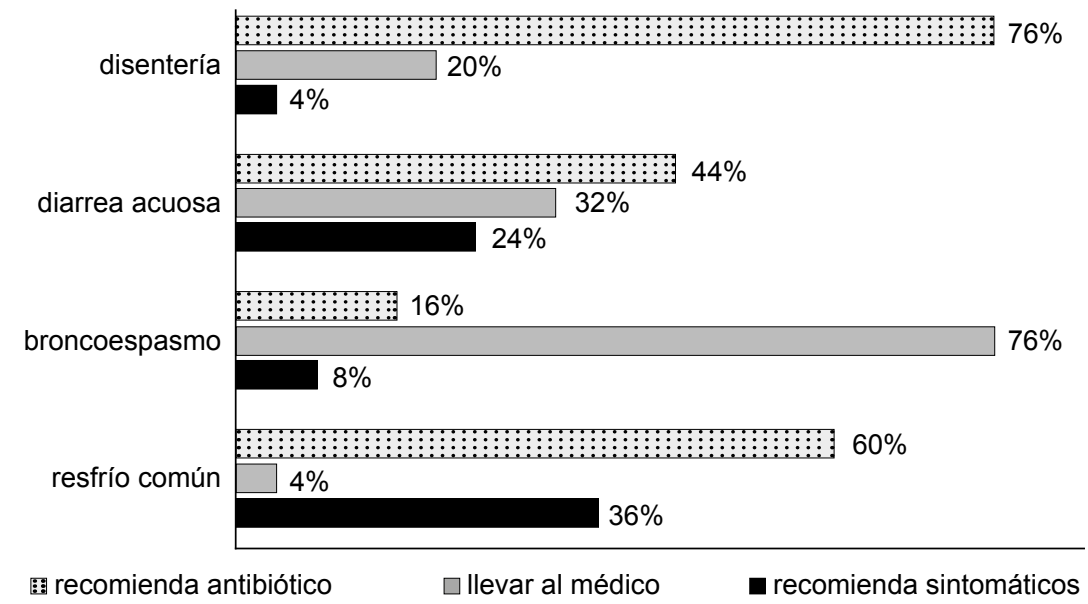

Figura 3. Recomendaciones por parte del farmacéutico para casos ficticios

uso de quinolonas se ha incrementado en cinco veces en todos los países estudiados (Argentina, Venezuela, Perú, México, Chile, Uruguay, Colombia y Brasil) ${ }^{(11)}$. Es por esto que no sorprende encontrar altos niveles de resistencia antibiótica en Perú ${ }^{(13-15,21-23)}$. En Latinoamérica es común poder conseguir antibióticos sin que medie una prescripción médica, debido a la carencia de leyes reguladoras que permiten su venta libre o por la falta de cumplimiento de las normas ${ }^{(18,24,25)}$.

En el Perú, a pesar de existir una regulación, es común adquirir antibióticos sin receta, tal como fue corroborado en nuestro estudio. Esto no solamente ocurre en Perú, en otros países de América del Sur como en Colombia y Chile se encontró que luego de años de establecida la norma de vender antibióticos solo con receta médica, su cumplimiento se diluye en el tiempo ${ }^{(11,18,24)}$.

Se han llevado a cabo intervenciones para disminuir el uso de antibióticos en algunos países, logrando disminuir la prescripción antibiótica tanto en países desarrollados como en países en vías de desarrollo ${ }^{(18,26,27)}$. Las intervenciones que promueven el uso apropiado de los antibióticos han demostrado alterar los patrones de resistencia favorablemente ${ }^{(28)}$. Sin embargo, en estudios previos han demostrado que la principal causa de uso inadecuado es la prescripción médica ${ }^{(1-3)}$.

Como lo muestra el presente estudio, los medicamentos que se compran con mayor frecuencia en una farmacia privada para tratar afecciones en niños menores de cinco años son, en su mayoría, antibióticos $(61,8 \%)$. Sin embargo, la mayoría de estas compras (87\%) es con una prescripción médica. Este hecho ya había sido descrito en estudios anteriores en nuestro medio, en donde el $90,8 \%$ del uso de antibióticos fue por prescripción del médico en un establecimiento de atención primaria, de ellas fueron innecesarias $83,1 \%$ de las prescripciones ${ }^{(1)}$. Por otro lado, ante casos hipotéticos en niños menores de cinco años, $77,1 \%$ de los médicos recomendarían

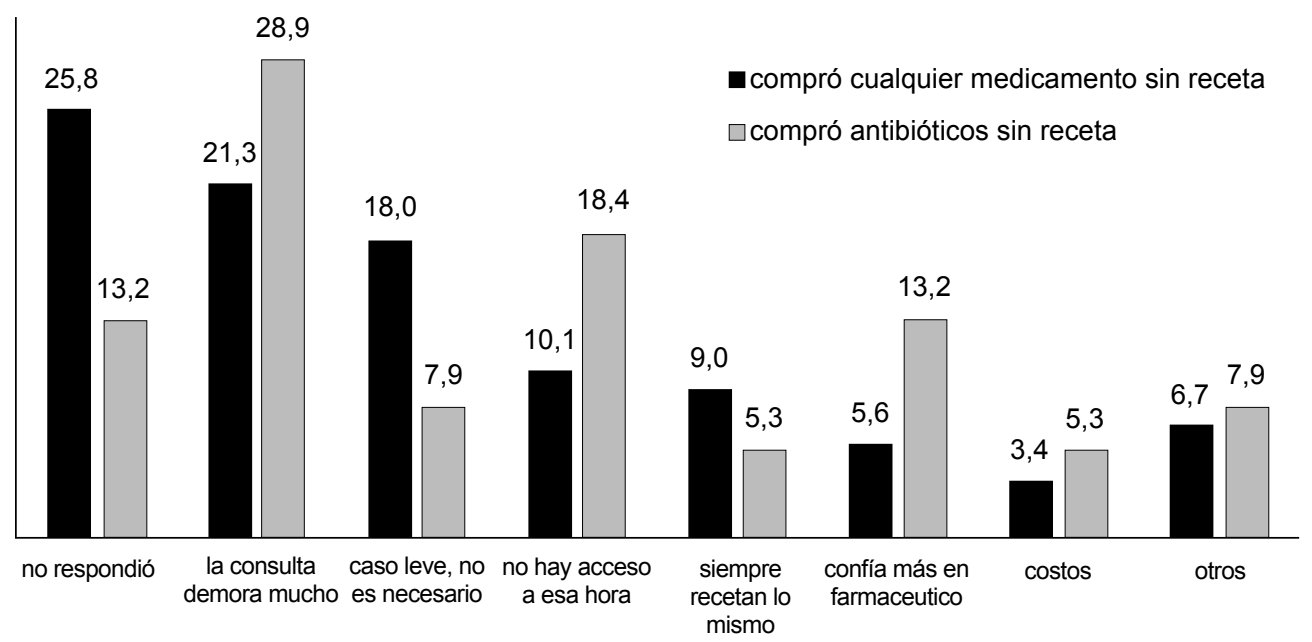

Figura 4. Causas por las que los padres o cuidadores compran sin receta médica 
un antibiótico en el caso de faringitis; 19,8\% usaría antibióticos en el caso de diarrea acuosa y $61,3 \%$ para tratar broncoespasmos ${ }^{(2)}$. Este es un problema grave que requiere una pronta intervención que debe incluir un adecuado programa de administración de los antibióticos, con medidas multidisciplinarias que incluyan, entre otras regulaciones, educación pasiva (guías de uso y capacitación), intervenciones (rondas clínicas, auditorías, reevaluación de las perspectivas de antibióticos, medidas que restrinjan su uso (formularios especiales, reporte de susceptibilidad microbiológica), así como medidas complementarias y consultorías.

En el presente estudio, dos tercios de los medicamentos comprados para tratar resfrío común y broncoespasmo fueron antibióticos, de los cuales más de la mitad fueron prescritos por el médico. Tomando en cuenta que en este grupo etario, las etiologías para estas afecciones son netamente de origen viral, los antibióticos son, en su mayoría, innecesarios. Asimismo, vemos que no solo se están utilizando antibióticos de primera línea para tratar afecciones respiratorias leves, también encontramos recetas que suelen reservarse para patógenos resistentes como lo son macrólidos, llegando a ser prescritos en un $7,2 \%$, y amoxicilina-ácido clavulánico en $2,2 \%$. No sorprende, entonces, que para afecciones de mayor intensidad, que sin embargo tampoco requieren prescripción antibiótica en este grupo, se incremente la prescripción antibiótica de segunda línea llegando a prescribirse macrólidos para tratar broncoespasmos en un $17,2 \%$ y para tratar faringitis en un $18,7 \%$.

Existe una tendencia en países en vías de desarrollo de incrementar el uso de antibióticos para tratar afecciones leves, aun cuando no hay signos de infección bacteriana ${ }^{(29,30)}$. Esto expone innecesariamente al niño a un antibiótico sin tener un efecto beneficioso para él y que, por el contrario, estaría incrementando la resistencia bacteriana, dejándonos sin armas futuras para un tratamiento de infecciones bacterianas que realmente lo necesiten.

Al presentar a los dispensadores de medicamentos casos ficticios que ocurren en niños menores de cinco años, en casi dos tercios de los casos ficticios de resfrío común y en casi la mitad de los casos de diarrea acuosa, el farmacéutico recomendó un antibiótico. Adicionalmente, cabe resaltar que la recomendación por parte de los farmacéuticos es un problema en sí, puesto que no preguntaban si el niño era alérgico a algún medicamento $(60,6 \%)$, no sabemos si es que recomendaron la dosis y duración de tratamiento adecuados para el peso y la edad del niño.

El estudio tiene limitaciones, es posible que haya influencia de la distancia de la farmacia o botica en relación al centro de salud, siendo posible que en las farmacias más alejadas de los centros de salud se vendan más antibióticos sin receta, o se pida más consejo a los farmacéuticos. Por otro lado, si bien se abarcaron diferentes horarios y días no laborables, no se abarcaron horarios por encima de las $20.00 \mathrm{~h}$. Asimismo, no se midió el consumo de antibióticos comprados por teléfono y entregados a domicilio.

En conclusión, en niños menores de 5 años, las madres o cuidadores prefieren buscar consejo médico antes de automedicar a sus hijos o buscar consejo del farmacéutico, como lo demuestra el presente estudio donde más del $80 \%$ de las compras de antibióticos en las farmacias fueron con receta médica. Sin embargo, existe un exceso de prescripción de antibióticos para tratar afecciones que no los requieren, y dada esta prescripción desde muy temprana edad, se acostumbra a los padres o cuidadores a usar antibióticos para tratar a sus hijos y esto podría llevar a una posterior automedicación, incrementando innecesariamente la resistencia bacteriana, como lo hemos determinado en un estudio previo ${ }^{(3)}$, en donde el uso sin receta médica se incrementó con la edad del niño y que padres o cuidadores "aprenden" a "reusar" recetas médicas conforme avanza la edad del niño por lo que es probable que cifras mucho más alarmantes se encuentren en grupos etarios de mayor edad. Este problema debe ser abordado de manera multidisciplinaria como una prioridad necesaria para disminuir el uso indiscriminado de antibióticos y la consecuente resistencia que este uso genera. Por otro lado, el personal de farmacia continúa recomendando medicamentos y antibióticos sin exigir receta, inclusive para su uso en niños.

Por lo tanto, la aplicación de intervenciones para disminuir el uso excesivo de antibióticos debería iniciarse en este grupo etario, enfocándose en la disminución de la prescripción médica. Sin dejar de lado la educación del personal que dispensa medicamentos en las farmacias o boticas, así como del fortalecimiento en la regulación de su venta con receta médica.

Contribuciones de autoría: LE participó en la concepción y diseño del estudio, recolección de datos, análisis e interpretación de datos, redacción del artículo, revisión crítica del artículo y aprobación de su versión final. TO participó en la obtención de financiamiento, concepción y diseño del estudio, recolección de datos, asesoría en la recolección de datos e interpretación de resultados y revisión crítica del artículo y aprobación de su versión final. MV, LJDV y JR en la obtención de financiamiento, concepción y diseño del estudio, y revisión crítica del artículo y aprobación de su versión final.

Fuentes de financiamiento: todas las fases de este estudio fueron financiadas por la Agencia Española de Cooperación Internacional (número de Grant : D/019499/08). JR está financiado por el programa I3 del Instituto de Salud Carlos III (España) program I3SNS, (número de Grant: CES11/012)

Conflictos de interés: los autores no tienen ningún conflicto de interés. 


\section{REFERENCIAS BIBLIOGRÁFICAS}

1. Ecker L, Olarte L, Vilchez G, Ochoa TJ, Amemiya I, Gil AI, et al. Physicians' responsibility for antibiotic use in infants from periurban Lima, Peru. Rev Panam Salud Publica. 2011;30(6):574-9.

2. Ecker L, Ochoa TJ, Vargas M, Del Valle LJ, Ruiz J. Preferences of antibiotic use in children less than five in physicians working health centers of primary level in peri-urban areas of Lima, Peru. Rev Peru Med Exp Salud Publica. 2013;30(2):181-9.

3. Ecker L, Ochoa TJ, Vargas M, Del Valle LJ, Ruiz J. Factors affecting caregivers' use of antibiotics available without a prescription in Peru. Pediatrics. 2013;131(6):e1771-9. doi: 10.1542/ peds.2012-1970.

4. Clavenna A, Sequi M, Bonati M. Differences in the drug prescriptions to children by Italian paediatricians and general practitioners. Eur J Clin Pharmacol. 2010;66(5):519-524. doi: 10.1007/s00228-010-0786-5.

5. Elshout G, Kool M, Van der Wouden JC, Moll HA, Koes BW, Berger MY. Antibiotic Prescription in Febrile Children: A Cohort Study during Outof-Hours Primary Care. J Am Board Fam Med. 2012;25(6):810-8. doi: 10.3122/jabfm.2012.06.110310.

6. Okeke IN, Laxminarayan R, Bhutta ZA, Duse AG, Jenkins P, O’Brien $\mathrm{TF}$, et al. Antimicrobial resistance in developing countries. Part I: recent trends and current status. Lancet Infect Dis. 2005;5(8):481-93. doi: 10.1016/ S1473-3099(05)70189-4

7. Okeke IN, Klugman KP, Bhutta ZA, Duse AG, Jenkins P, O'Brien TF, et al. Antimicrobial resistance in developing countries. Part II: strategies for containment. Lancet Infect Dis. 2005;5(9):568-80. doi: 10.1016/ S1473-3099(05)70217-6

8. Bartoloni A, Pallecchi L, Fiorelli C, Di Maggio T, Fernandez C, Villagran AL, et al. Increasing resistance in commensal Escherichia coli, Bolivia and Peru. Emerg Infect Dis. 2008;14(2):338-40. doi: 10.3201/eid1402.070138.

9. Bartoloni A, Pallecchi L, Rodriguez H, Fernandez C, Mantella A, Bartalesi $\mathrm{F}$, et al. Antibiotic resistance in a very remote Amazonas community. Int $\mathrm{J}$ Antimicrob Agents. 2009;33(2):125-9. doi: 10.1016/j.ijantimicag.2008.07.029.

10. Roca I, Akova M, Baquero F, Carlet J, Cavaleri M, Coenen S, et al. The global threat of antimicrobial resistance: science for intervention. New Microbes and New Infect. 2015;6:22-9. doi: 10.1016/j.nmni.2015.02.007.

11. Wirtz VJ, Dreser A, Gonzales R. Trends in antibiotic utilization in eight Latin American countries, 1997-2007. Rev Panam Salud Publica. 2010;27(3):219-25.

12. Jones RN, Guzman-Blanco M, Gales AC, Gallegos B, Castro AL, Martino $\mathrm{MD}$, et al. Susceptibility rates in Latin American nations: report from a regional resistance surveillance program (2011). Braz J Infect Dis. 2013;17(6):672-81. doi: 10.1016/j. bjid.2013.07.002.

13. Ochoa TJ, Ruiz J, Molina M, Del Valle LJ, Vargas M, Gil AI, et al. High frequency of antimicrobial drug resistance of diarrheagenic Escherichia coli in infants in Peru. Am J Trop Med Hyg. 2009;81(2):296-301.

14. Pons MJ, Mosquito S, Gomes C, Del Valle LJ, Ochoa TJ, Ruiz J. Analysis of quinolone-resistance in commensal and diarrheagenic Escherichia coli isolates from infants in Lima, Peru. Trans R Soc Trop Med Hyg. 2014;108(1):22-8. doi: $10.1093 / \mathrm{trstmh} / \mathrm{trt} 106$.

15. Pons MJ, Mosquito $S$, Ochoa TJ, Vargas M, Molina M, Lluque A, et al. Levels of quinolones resistance and other antimicrobial in non-pathogenic Escherichia coli strains in children from the periurban area of Lima, Peru. Rev Peru Med Exp Salud Publica. 2012;29(1):82-6.

16. Hart CA, Kariuki S. Antimicrobial resistance in developing countries. BMJ. 1998;317(7159):647-50.

17. Wolff MJ. Use and misuse of antibiotics in Latin America. Clin Infect Dis. 1993;17 Suppl 2:S346-51.

18. Bavestrello FL, Cabello MA. Community antibiotic consumption in Chile, 2000-2008. Rev Chilena Infectol. 2011;28(2):107-12. doi: / S0716-10182011000200001.

19. Dameh M, Green J, Norris P. Overthe-counter sales of antibiotics from community pharmacies in Abu Dhabi. Pharm World Sci. 2010;32(5):643-50. doi: 10.1007/s11096-010-9418-5.

20. Paphassarang C, Philavong K, Boupha $B$, Blas E. Equity, privatization and cost recovery in urban health care: the case of Lao PDR. Health Policy Plan. Dec 2002;17 Suppl:72-84.

21. Ochoa TJ, Mohr J, Wanger A, Murphy JR, Heresi GP. Community-associated methicillin-resistant Staphylococcus aureus in pediatric patients. Emerg Infect Dis. 2005;11(6):966-8.

22. Ochoa TJ, Rupa R, Guerra $H$, Hernandez H, Chaparro E, Tamariz $\mathrm{J}$, et al. Penicillin resistance and serotypes/serogroups of Streptococcus pneumoniae in nasopharyngeal carrier children younger than 2 years in Lima, Peru. Diagn Microbiol Infect Dis. 2005;52(1):59-64.

23. Torres N, Velasquez R, Mercado EH, Egoavil M, Horna G, Mejía L, et al. Antibiotic resistance of streptococcus pneumoniae among healthy nasopharyngeal carriers in seven regions of Peru. Rev Peru Med Exp Salud Publica. 2013;30(4):575-82.

24. Vacca CP, Nino CY, Reveiz L. [Restriction of antibiotic sales in pharmacies in Bogota, Colombia: a descriptive study]. Rev Panam Salud Publica. 2011;30(6):586-91.

25. Organización Panamericana de la Salud. Legislación sobre antibióticos en america latina. Washinton, DC.: OPS; 2004.

26. Grigoryan L, Monnet DL, HaaijerRuskamp FM, Bonten MJ, Lundborg $S$, Verheij TJ. Self-medication with antibiotics in Europe: a case for action. Curr Drug Saf. 2010;5(4):329-32.

27. McNulty CA, Francis NA. Optimizing antibiotic prescribingin primarycaresettings in the UK: findings of a BSAC multidisciplinary workshop 2009. J Antimicrob Chemother. 2010;65(11):2278-84. doi: $10.1093 / \mathrm{jac} / \mathrm{dkq} 361$.

28. Arnold SR, Straus SE. Interventions to improve antibiotic prescribing practices in ambulatory care. Cochrane Database Syst Rev. 2005;19(4):CD003539.

29. Calva J, Bojalil R. Antibiotic use in a periurban community in Mexico: a household and drugstore survey. Soc Sci Med. 1996;42(8):1121-8.

30. Nakajima R, Takano T, Urnaa V, Khaliun N, Nakamura K. Antimicrobial use in a country with insufficient enforcement of pharmaceutical regulations: A survey of consumption and retail sales in Ulaanbaatar, Mongolia. South Med Review. 2010;3(1):19-23.

Correspondencia: Lucie Ecker

Dirección: Instituto de Investigación Nutricional, Av. La Molina 1885, La Molina,

Lima-Perú, Teléfono (+511) 3496023

Correo electrónico:lecker@iin.sld.pe 